\title{
In vivo derivation of glucose- competent pancreatic endocrine cells from bone marrow without evidence of cell fusion
}

\author{
Andreea Ianus, ${ }^{1}$ George G. Holz, ${ }^{2,3}$ Neil D. Theise, ${ }^{1}$ and Mehboob A. Hussain ${ }^{3,4}$ \\ ${ }^{1}$ Department of Pathology, \\ ${ }^{2}$ Department of Physiology and Neuroscience, \\ ${ }^{3}$ Department of Pharmacology, and \\ ${ }^{4}$ Department of Medicine, New York University School of Medicine, New York, New York, USA
}

\begin{abstract}
Bone marrow harbors cells that have the capacity to differentiate into cells of nonhematopoietic tissues of neuronal, endothelial, epithelial, and muscular phenotype. Here we demonstrate that bone marrow-derived cells populate pancreatic islets of Langerhans. Bone marrow cells from male mice that express, using a CRE-LoxP system, an enhanced green fluorescent protein (EGFP) if the insulin gene is actively transcribed were transplanted into lethally irradiated recipient female mice. Four to six weeks after transplantation, recipient mice revealed $Y$ chromosome and EGFP double-positive cells in their pancreatic islets. Neither bone marrow cells nor circulating peripheral blood nucleated cells of donor or recipient mice had any detectable EGFP. EGFP-positive cells purified from islets express insulin, glucose transporter 2 (GLUT2), and transcription factors typically found in pancreatic $\beta$ cells. Furthermore, in vitro these bone marrow-derived cells exhibit - as do pancreatic $\beta$ cells - glucose-dependent and incretin-enhanced insulin secretion. These results indicate that bone marrow harbors cells that have the capacity to differentiate into functionally competent pancreatic endocrine $\beta$ cells and that represent a source for cell-based treatment of diabetes mellitus. The results generated with the CRE-LoxP system also suggest that in vivo cell fusion is an unlikely explanation for the "transdifferentiation" of bone marrow-derived cells into differentiated cell phenotypes.
\end{abstract}

J. Clin. Invest. 111:843-850 (2003). doi:10.1172/JCI200316502.

\section{Introduction}

Diabetes mellitus results when there is an inadequate functional mass of pancreatic $\beta$ cells. In type 1 diabetes, immune-mediated destruction of $\beta$ cells leaves a markedly reduced $\beta$ cell mass. In type 2 diabetes, there is an increased demand for secreted insulin in the face of nearly normal, but insufficient and not increased, $\beta$ cell mass. With the worldwide prevalence of diabetes increasing rapidly, considerable interest has developed in finding mechanisms to increase $\beta$ cell mass by stimulating endogenous regeneration of islets (1-7) or by transplanting donor islets (8) or in vitro-differentiated islet-like cells $(9,10)$. To this end, regeneration of pancreatic endocrine cells has been

Received for publication July 25, 2002, and accepted in revised form February 11, 2003.

Address correspondence to: Mehboob A. Hussain,

Departments of Medicine and Pharmacology,

New York University School of Medicine,

550 First Avenue, MSB 424, New York,

New York 10016, USA.

Phone: (212) 263-0942; Fax: (212) 263-7133;

E-mail: hussam02@popmail.med.nyu.edu.

Conflict of interest: The authors have declared that no conflict of interest exists.

Nonstandard abbreviations used: glucose transporter 2

(GLUT2); enhanced green fluorescent protein (EGFP). demonstrated in several experimental models (1-7). Multipotent stem cells have been described within pancreatic islets $(11,12)$ and in nonendocrine compartments of the pancreas $(9,13)$, and these cells have the capacity to differentiate into pancreatic islet-like structures in vitro. Furthermore, cells that do not reside within the pancreas, such as hepatic oval cells $(14,15)$ and embryonic stem cells, have been differentiated into pancreatic endocrine hormone-producing cells in vitro (16) and in vivo (17). The latter can correct the diabetes phenotype in mice (17).

After transplantation of bone marrow or enriched hematopoietic stem cells, skeletal lung, gut, and skin epithelia (18), myoblasts $(19,20)$, cardiac myoblasts $(21,22)$, endothelium $(23,24)$, hepatic and biliary duct epithelium $(14,25,26)$, or neuroectodermal cells $(27$, 28 ) of donor origin can be found in the recipient animals. This phenomenon of engraftment of bone marrow-derived cells into multiple tissues is also found in humans $(29,30)$. Thus, adult bone marrow harbors cells that have pluripotent differentiation capacity. Recently, pluripotent mesenchymal stem cells in adult bone marrow have been described that have the capacity to differentiate into mesenchymal lineage cells, endothelium $(31,32)$, and endoderm tissue (33) in vitro and in vivo (34). However, most studies on bone 
marrow-derived differentiated cells are limited to morphological characteristics and gene and protein expression profiling of these cells. With the exception of cardiac and liver engraftment $(21,33)$, there is little information on the functional capability of bone marrow-derived cells that engraft into extramedullary tissues. Furthermore, the paradigm of bone marrow cells "transdifferentiating" into multiple cell lineages has been challenged by the observation that, in vitro, pluripotent cells fuse with differentiated cells and thus adapt a "differentiated" phenotype $(35,36)$.

In the present study we have used the CRE-LoxP system (37), which allows identification, purification, and functional characterization of insulin-expressing cells from pancreatic islets that have been derived in vivo from transplanted bone marrow. These cells express insulin, glucose transporter 2 (GLUT2), and transcription factors typically found in pancreatic $\beta$ cells and lack the stem cell marker oct-4. These bone marrow-derived cells secrete insulin in response to a glucose stimulus or to the incretin hormone analog exendin-4. Furthermore, the results also suggest that cell fusion $(35,36)$ is in vivo an unlikely explanation for the "transdifferentiation" of bone marrow-derived cells adapting the pancreatic $\beta$ cell phenotype.

\section{Methods}

Murine model of bone marrow transplantation and experimental design. Animals were maintained under conditions approved by the institutional Animal Care and Use Committee at New York University School of Medicine. Transgenic mice expressing the phage CRE recombinase (CRE) under control of the rat insulin II promoter (INS2-CRE) (38) were obtained from The Jackson Laboratory (Bar Harbor, Maine, USA; C57BL/6 background). Mice expressing enhanced green fluorescent protein (EGFP) at the ROSA26 locus (39) preceded by three floxed translation stop codons (ROSA-stoplox-EGFP; C57BL/6 background) (40) were obtained from $\mathrm{G}$. Eberl and D. Littman (New York University School of Medicine). The stop codons are removed by activity of the CRE that recognizes the LoxP sequence and removes the chromosomal portion flanked by the LoxP sites (i.e., translation stop sequences), thus resulting in a permanent expression of EGFP (37). To generate INS2*EGFP mice, heterozygous INS2-CRE mice were crossed with hemizygous ROSA-stoplox-EGFP mice, and offspring were genotyped at the time of weaning. In INS2*EGFP mice, cells that activate the insulin promoter express $\mathrm{CRE}$ and become identifiable by their expression of EGFP. Genotyping was performed with the following primers according to standard protocols: EGFP (forward, 5'-GCGAGGGCGATGCCACCTACGGCA-3'; reverse, 5'-GGGTGTTCTGCTGGTAGTGGTCGG-3'; 450 bp), CRE (forward, 5'-TAAAGATATCTCACGTACTGACGG-3'; reverse, $5^{\prime}$-TCTCTGACCAGAGTCATCCTTAGC-3'; 250 bp).

Bone marrow from male donor mice was flushed under sterile conditions with HBSS from the medullary cavities of tibiae and femurs using a 25 -gauge needle.
Marrow cells were checked for viability with the trypan blue method and filtered (Nitex 03-50/31; Sefar America, Kansas City, Missouri, USA) as previously described (18). Recipients were irradiated female mice (1,050-1,100 cGy from a Gammacell 40 small-animal irradiator; Atomic Energy of Canada, Kanata, Ontario, Canada) and received approximately $10^{6}$ unfractionated bone marrow-derived cells by tail vein injection. Recipient mice were kept in sterile cages with sterile chow and water for 2 weeks after irradiation and euthanized at 4-6 weeks after bone marrow transplantation for tissue harvesting. Because all animals had the same C57BL/ 6 background, no alloimmune or graft-versushost response was expected (nor observed). We checked engraftment by determining the fraction of peripheral blood nucleated cells with a Y chromosome (two counts of 100 nucleated cells per animal).

Experimental bone marrow transplantation procedures are summarized in Figure 1. In one set of experiments, donor animals were male INS2*EGFP mice, and recipients were female wild-type mice (experiment 1). In a second set of experiments, donor animals were male INS2-CRE mice, and recipients were female ROSA-EGFP mice (experiment 2). Experiment 2 tested for EGFP expression in recipient cells secondary to any cell fusion with donor cells expressing CRE (see Discussion).

Islet cell isolation. Islets were isolated using the collagenase method as previously described (41). Briefly, the pancreas was injected with $1 \mathrm{ml}$ of collagenase $\mathrm{P}$ (2 $\mathrm{mg} / \mathrm{ml}$; Roche Diagnostics Corp., Indianapolis, Indiana, USA) and DNaseI $(0.5 \mathrm{mg} / \mathrm{ml}$; Roche Diagnostics Corp.) and incubated at $37^{\circ} \mathrm{C}$ in a total of $10 \mathrm{ml}$ of digestion solution under constant shaking and intermittent vortexing. Islets were subsequently washed several times in HBSS with BSA $(5 \mathrm{mg} / \mathrm{ml})$ and handpicked under a dissecting microscope. Islets were dispersed into single cells by suspension in trypsin EDTA (GIBCO BRL; Life Technologies Inc., Grand Island, New York, USA) and trituration through a siliconized Pasteur pipette. Cells were then cultured in RPMI 1640 (GIBCO BRL; Life Technologies Inc.), 5.5 $\mathrm{mM}$ glucose, $10 \% \mathrm{FCS}$ in a humidified incubator $(95 \%$ air, $5 \% \mathrm{CO}_{2}$ ) at $37^{\circ} \mathrm{C}$.

EGFP-positive cells were isolated from EGFP-negative cells by FACS (Becton Dickinson and Co., Franklin Lakes, New Jersey, USA) or manual picking under a fluorescent microscope for further culture, RT-PCR (see below), or immunostaining and FISH. FACS data are given in Figure 4 and were generated on WinMDI 2.8 software (Joseph Trotter, Scripps Institute FACS core facility server, La Jolla, California, USA). Units of $x$ and $y$ axes are arbitrary but kept the same for all experiments. Parts a, e, and i of Figure 4 have linear axes and show forward and side scatter of cells. Parts $\mathrm{b}-\mathrm{d}, \mathrm{f}-\mathrm{h}$, and $\mathrm{j}-\mathrm{l}$ have $x$ and $y$ axes in logarithmic scale and show green fluorescence intensity in the $x$ axis (EGFP signal) and phycoerythrin (red) fluorescence intensity in the $y$ axis (phycoerythrin signal). 


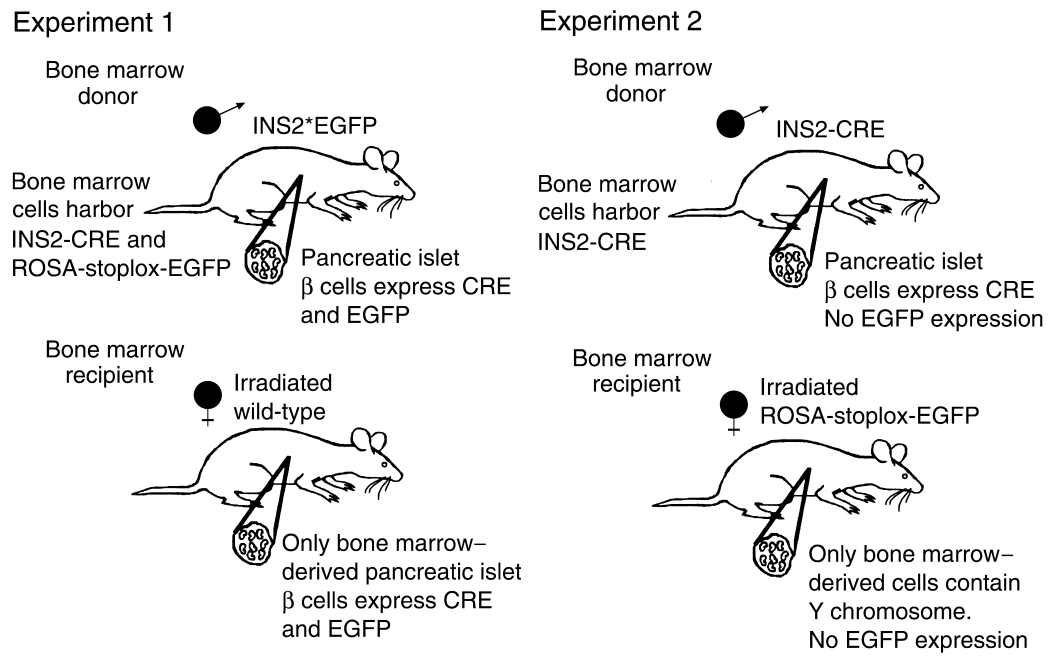

Figure 1

Mouse bone marrow transplantation protocol. Experiment 1: Bone marrow from male INS2*EGFP mice was injected into irradiated female wild-type mice. Experiment 2: Bone marrow from male INS2-CRE mice was injected into irradiated female ROSA-stoplox-EGFP mice.

Immunobistochemistry and FISH. Tissues were fixed in $4 \%$ paraformaldehyde for 2 hours at room temperature and subsequently in $30 \%$ sucrose overnight at $4^{\circ} \mathrm{C}$ before embedding in Tissue-Tek OCT compound (Sakura Finetek USA Inc., Torrance, California, USA) for sectioning $(5-7 \mu \mathrm{m})$. A total of 200 pancreatic sections were analyzed. Isolated islet cells were fixed on microscope slides with $4 \%$ paraformaldehyde for 4 minutes at room temperature. Tissue sections and cells were treated with $100 \%$ methanol for 2 minutes at $-20^{\circ} \mathrm{C}$ and then blocked $3 \%$ with normal donkey serum for 10 minutes at room temperature. Samples were then incubated at $4^{\circ} \mathrm{C}$ overnight with nonimmune serum, a guinea pig antiserum raised against insulin (Linco Research Inc., St. Charles, Missouri, USA) at a dilution of 1:1,000, rabbit anti-IPF-1 $(1: 500)(41)$, or rabbit anti-HNF3 $\beta(1: 2,000)$ (provided by T. Jessel, Columbia University, New York, New York, USA; and A. Ruiz i Altaba, New York University School of Medicine). After rinsing with PBS, slides were blocked again with $3 \%$ donkey serum for 10 minutes at room temperature and then localized with donkey anti-guinea pig or donkey anti-rabbit with conjugated rhodamine X (Jackson ImmunoResearch Laboratories Inc., West Grove, Pennsylvania, USA) (1:500) for 30 minutes at room temperature. Slides were mounted in fluorescent mounting medium (Vector Laboratories Inc., Burlingame, California, USA) and stored in the dark. For frozen sections, a rabbit anti-green fluorescent protein IgG conjugated with Alexa Fluor 488 (Molecular Probes Inc., Eugene, Oregon, USA) at a dilution of 1:2,000 was used to detect the presence of EGFP-positive cells. After image capturing, the same samples were analyzed for the presence of mouse Y chromosome with FISH
(Rainbow*FISH; Cambio Ltd., Cambridge, United Kingdom) according to the manufacturer's recommendation. A digestion with proteinase $\mathrm{K}\left(10 \mu \mathrm{g} / \mathrm{ml}\right.$ at $37^{\circ} \mathrm{C}, 5$ minutes $)$ (Sigma-Aldrich, St. Louis, Missouri, USA) was added at the beginning of the FISH protocol. Nuclei were stained with 4',6-diamidine-2'-phenylindole dihydrochloride (DAPI) using fluorescent mounting medium containing DAPI at $1.5 \mu \mathrm{g} / \mathrm{ml}$ (Vector Laboratories Inc.).

Imaging was performed on a Zeiss Axioskop 2 fluorescent microscope (Carl Zeiss MicroImaging Inc., Thornwood, New York, USA) equipped with a cooled charge-coupled device digital camera (Hamamatsu Photonics K.K., Hamamatsu City, Japan) and Improvision Open Lab software (Improvision Inc., Lexington, Massachusetts, USA) that allows pseudocoloring. Images were captured using the appropriate light absorption and emission filters supplied by the manufacturer of the microscope.

Reverse transcription and PCR. A subset of fluorescence-sorted islet cells were taken in culture for 24 hours and then hand-picked twice for positive EGFP fluorescence. One hundred of these cells were used for RT-PCR analysis. Total RNA from cells was purified using RNeasy with an RNase-free DNase digestion step (QIAGEN Inc., Valencia, California, USA). Reverse transcription was performed with Omniscript (QIAGEN Inc.), and PCR was performed using recombinant DNA polymerase (Takara Shuzo Co., Ltd., Shiga, Japan). Primers and PCR product size were as follows: insulin I (forward, 5'-TAGTGACCAGCTATAATCAGAG-3'; reverse, 5'-ACGCCAGGTCTGAAGGTCC-3'; 288 bp), insulin II (forward, 5'-CCCTGCTGGCCCTGCTCTT-3'; reverse, 5' AGGTCTGAAGGTCACCTGCT-3'; 212 bp), IPF-1 (forward, 5'-TGTAGGCAGTACGGGTCCTC-3'; reverse, 5'CCACCCCAGTTTACAAGCTC-3'; 325 bp), HNF3 $\beta$ (forward, 5'-ACCTGAGTCCGAGTCTGACC-3'; reverse, 5'GGCACCTTGAGAAAGCAGTC-3'; 345 bp), HNF1 $\alpha$ (forward, 5'-TCACAGACACCAACCTCAGC-3'; reverse, 5'-GAGGACACTGTGGGACTGGT-3'; 202 bp), HNF1 $\beta$ (forward, 5'-CCTAGGCTCCAACTTGGTCA-3'; reverse, 5'-TGTAGCGCACTCCTGACATC-3'; 203 bp), PAX6 (forward, 5'AAGAGTGGCGACTCCAGAAGTTG-3'; reverse, 5' -ACCACACCTGTATCCTTGCTTCAGG-3'; 545 bp), CD45 (C57BL/6) (forward, 5'-AACTGGAACACTGCCTGCTT-3'; reverse, 5'-GACCACCTCACTGTCACGTTT-3'; 198 bp), oct-4 (forward, 5'-GGCGTTCTCTTTGGAAAGGTGTTC-3'; reverse, 5'-CTCGAACCACATCCTTCTCT-3'; 293 bp), cyclophilin (forward, 5'-CAGACGCCACTGTCGCTTT-3'; reverse, 5'-TGTCTTTGGAACTTTGTCTGCAA-3'; 132 bp). An oct-3/4 control plasmid was a generous gift from $L$. Dailey (New York University School of Medicine). 

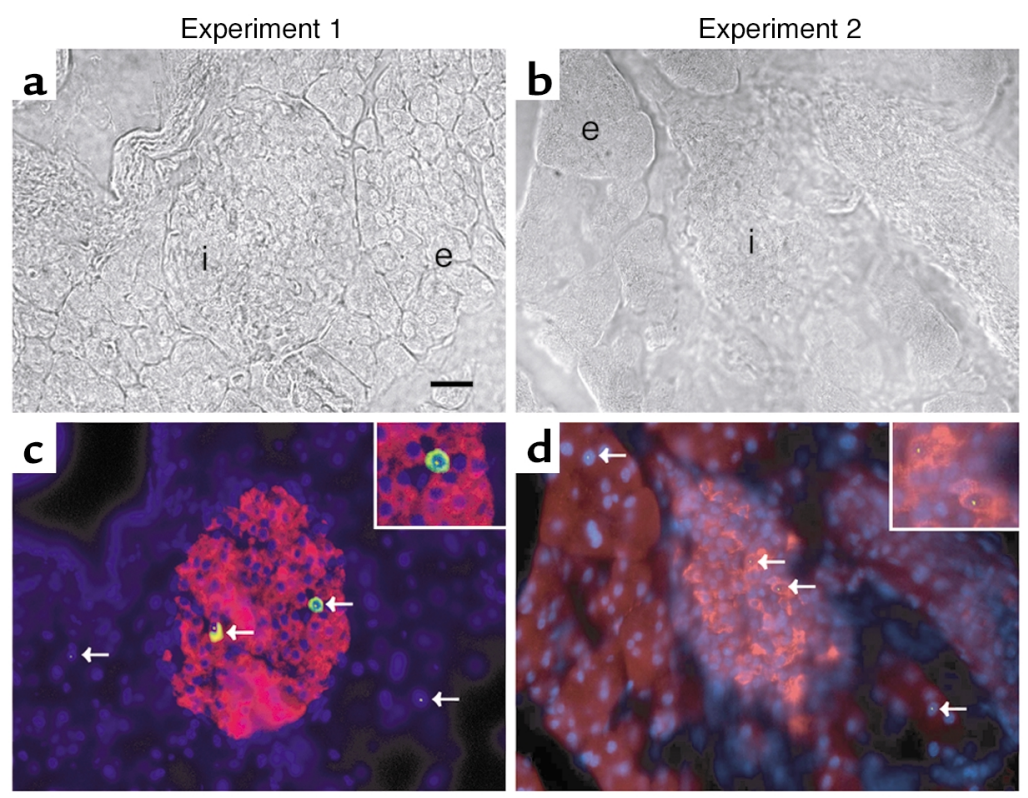

\section{Figure 2}

Immunofluorescence and FISH for $\mathrm{Y}$ chromosome of pancreatic 5- to $7-\mu \mathrm{m}$ frozen sections from recipient mice of experiments 1 and 2 (see Figure 1). (a and b) Bright-field phase images. (c and d) Composite overlay image of immunofluorescence for insulin (red) and EGFP (green), and FISH for Y chromosome (yellow) and DAPI staining of nuclei (blue). Y chromosome-positive cells (arrows) are present in the islet (i) and in the exocrine portions of the pancreas (e). EGFP is present only in insulin-positive cells in the islet.

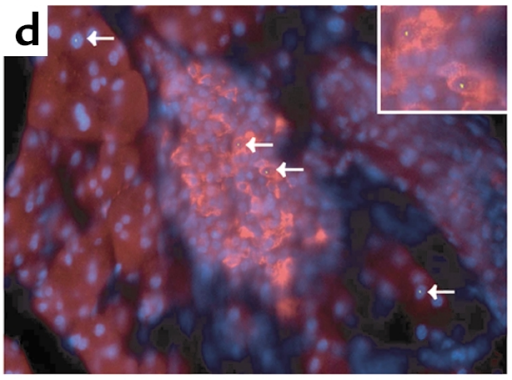
Scale bar, $10 \mu \mathrm{m} ; \times 400$. Insets in $\mathbf{c}$ and $\mathbf{d}$ are magnified areas of EGFP and insulin double-positive cells containing a $Y$ chromosome.

Functional characterization of islet cells: insulin secretion. After FACS sorting and/or manual picking, EGFP-positive islet cells (1,000 cells per microtiter well) were cultured for 24 hours before further analysis. Cells were then switched to medium containing either $5.5 \mathrm{mM}$ or $11.1 \mathrm{mM}$ glucose for an additional 10 hours. Some cells were also stimulated with the glucagon-like peptide- 1 analog exendin- 4 (Sigma-Aldrich; $10 \mathrm{nM}$ ) for the last 4 hours of incubation in $11.1 \mathrm{mM}$ glucose. As control cells, isolated islet cells from INS2*EGFP mouse were taken for a parallel assay. Supernatant of islet cells was harvested, gently spun, and analyzed for insulin by ELISA (Ultra sensitive Insulin ELISA Kit; Crystal Chem Inc., Chicago, Illinois, USA).

\section{Results}

We report on experiments 1 and 2 (Figure 1), conducted in parallel three times each with consistent results. A total of 48 animals were irradiated and injected with bone marrow cells. We observed an approximately $30 \%$ mortality rate in the first week after irradiation. Results of 21 animals in experiment 1 and 10 animals in experiment 2 are shown. Engraftment was found to be $70-90 \%$ when peripheral blood nucleated cells were examined for the presence of a Y chromosome.

On histological examination, we did not detect any changes suggestive of radiation injury. When wildtype female recipient animals were transplanted with bone marrow cells from male INS2*EGFP mice, EGFP-positive cells were detected within the islets of recipient mice (Figure 2 ) at 4-6 weeks after transplantation. On frozen sections (Figure 2) as well as in single-cell preparations (Figure 3, a-d), these EGFPpositive cells expressed insulin, as determined by
Insulin

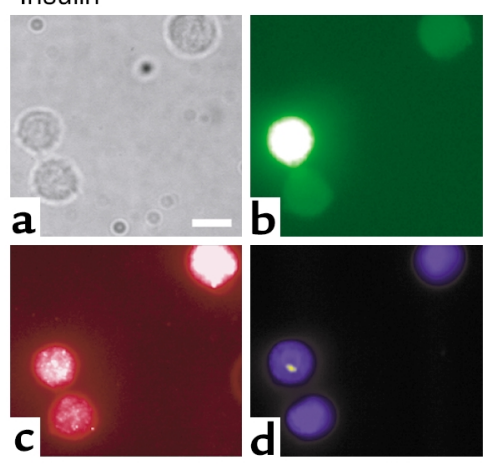

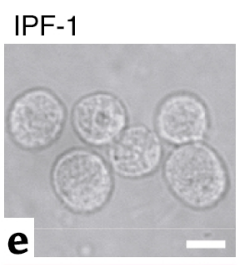

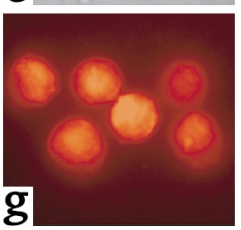

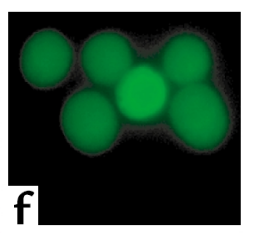

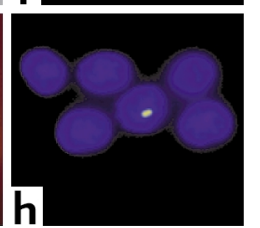

HNF3 $\beta$

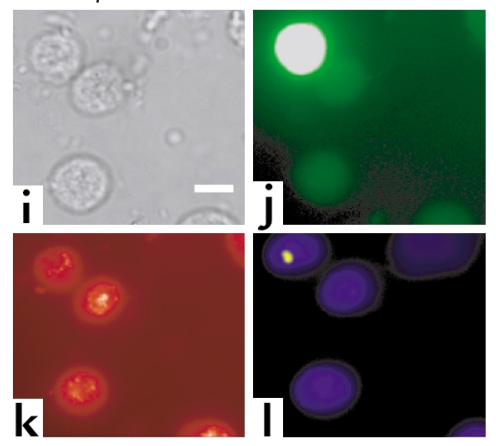

Figure 3

Immunofluorescence and FISH of isolated, dispersed pancreatic islet cells from experiment 1 (see Figure 1). Images of identical fields are shown in the respective panels: (a-d) insulin, (e-h) IPF-1, and (i-I) HNF3 $\beta$ immunodetection. (a, e, and $\mathbf{i})$ Bright-field phase; (b, $\mathbf{f}$, and $\mathbf{j}$ ) EGFP (note slight autofluorescence of control isolated islet cells); (c, $\mathbf{g}$, and $\mathbf{k}$ ) Immunostaining with rhodamine X-labeled secondary antibody for panels. (c) Insulin, (g) IPF-1, (k) HNF3 $\beta$, and (d h, and I) FISH for Y chromosome (in yellow) and nucleus stain with DAPI (blue). $\mathrm{Y}$ chromosome is present only in EGFP-positive cells. Scale bar, $5 \mu \mathrm{m} ; \times 630$. 

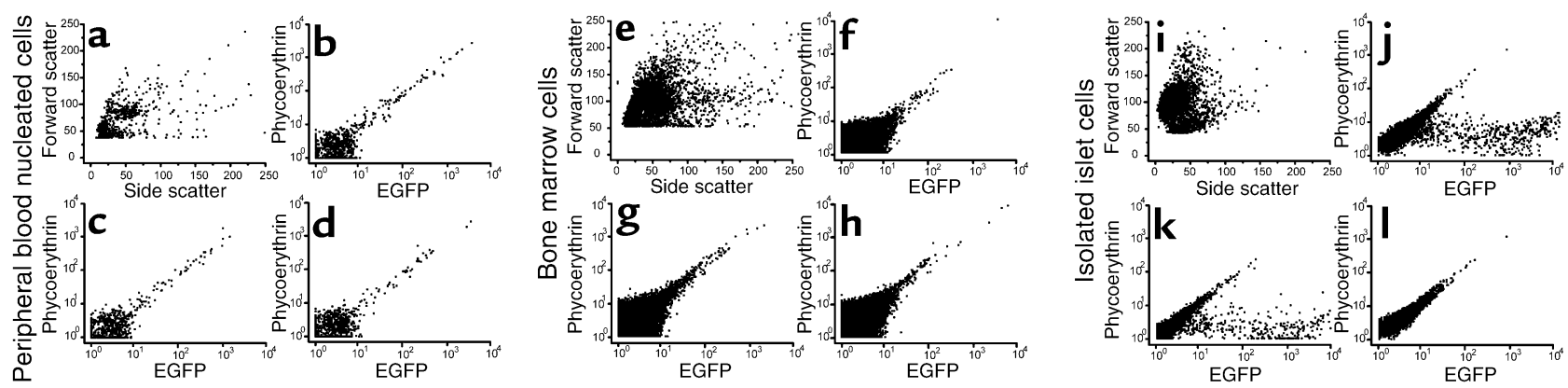

Figure 4

Cell sorting and fluorescence analysis. (a-d) Peripheral blood nucleated cells; (e-h) bone marrow cells; (i-I) isolated and dispersed islet cells. $(\mathbf{a}, \mathbf{e}$, and $\mathbf{i})$ Representational scatter plot of side ( $x$ axis) and forward $(y$ axis) scatter of respective cells. (b-d, $\mathbf{f}-\mathbf{h}$, and $\mathbf{j}-\mathbf{l})$ Fluorescence scans ( $x$ axis, EGFP; $y$ axis, phycoerythrin [red] filter) of respective cells. (b, f, and $\mathbf{j}$ ) Cells from INS*EGFP donor mice (experiment 1$)$. (c, $\mathbf{g}$, and $\mathbf{k}$ ) Cells from irradiated wild-type mice transplanted with bone marrow from INS*EGFP mice (experiment 1 ). (d, $\mathbf{h}$, and I) Cells from irradiated ROSA-stoplox-EGFP mice transplanted with bone marrow from INS2-CRE mice (experiment 2). No fluorescence is detectable in donor or recipient peripheral blood nucleated cells or bone marrow cells. EGFP is detectable in islets of donor animals of experiment 1 and in the recipients of experiment 1 . In $\mathbf{b}-\mathbf{d}, \mathbf{f}-\mathbf{h}$, and $\mathbf{j}-\mathbf{l}$, cells in the line equidistant from both axes show some autofluorescence, and cells in the lower right quadrant have EGFP signal. See Figure 1 for description of experiments 1 and 2 .

immunohistochemistry. Furthermore, on FISH analysis, these cells revealed a $\mathrm{Y}$ chromosome within their nuclei, indicating that these cells were derived from the bone marrow of the male donor mouse (Figures 2 and 3). Bone marrow-derived cells from islets expressed not only insulin, but also transcription factors specific for $\beta$ cells (IPF-1; Figure 3 , e-h) and endoderm (HNF3 $\beta$; Figure 3, i-l). In contrast, when ROSA-stoplox-EGFP female mice received bone marrow transplantation from INS2-CRE male donor mice (experiment 2 ), none of the cells within islets or the rest of the pancreas were fluorescent (Figure 2, b and d). Nevertheless, Y chromosome-positive cells were detectable within the islets, indicating that, in experiment 2 , engraftment of bone marrow-derived cells did take place in the islet; no recombination events at the LoxP sites of recipient mice were detectable as assessed by absent immunostaining for
EGFP (Figure 2d). In contrast, after we forced fusion in vitro (42) between INS2-CRE bone marrow cells and ROSA-stoplox-EGFP islet cells, we detected fluorescence (data not shown). These results indicate that in vivo cell fusion is unlikely to underlie apparent "transdifferentiation" of bone marrow-derived cells into a $\beta$ cell phenotype.

Fluorescence-activated cell analysis $\left(10^{4}\right.$ cells counted per islet cell preparation) (Figure 4) and/or manual counting (300 cells counted) showed that, 4-6 weeks after bone marrow transplantation, the proportion of EGFP-expressing cells within the islet ranged from $1.7 \%$ to $3 \%$ of all cells within an islet, as compared with $80 \%$ to $85 \%$ in islets from control INS2*EGFP mice. Neither peripheral blood nucleated cells (Figure 4, a-d) nor bone marrow (Figure 4, e-h) from donor nor recipient mice revealed detectable EGFP in any of the experiments.

\section{Figure 5}

RT-PCR of isolated and FACS-sorted cells from experiment 1. (a) Peripheral blood nucleated cells (PBNCs) of recipient mice express cyclophilin (lane 1 ) as well as CD45 (lane 2), and EGFP-expressing cells derived from pancreatic islets express cyclophilin (lane 3 ), lack CD45 (lane 4), and express insulin (lane 5). (b) EGFPexpressing cells isolated from pancreatic islets do not express the stem cell marker oct-3/4. Lane 1: Positive control oct-3/4 plasmid. Bone marrow cells express cyclophilin (lane 2) and oct-3/4 (lane 3), and EGFPexpressing cells from pancreatic islets express cyclophilin (lane 4) but not oct-3/4 (lane 5). (c) EGFP-expressing cells isolated from pancreatic islets express cyclophilin (lane 1), insulin I (lane 2), IPF-1 (lane 4), and HNF3 $\beta$ (lane 5). Lane 3 is empty. (d) EGFP-expressing cells derived from pancreatic islets express cyclophilin (lane 1), insulin II (lane 2), insulin I (lane 3), GLUT2 (lane 4), HNF1 $\alpha$ (lane 5), HNF1 $\beta$ (lane 6), and PAX6 (lane 7). $\mathbf{a}$
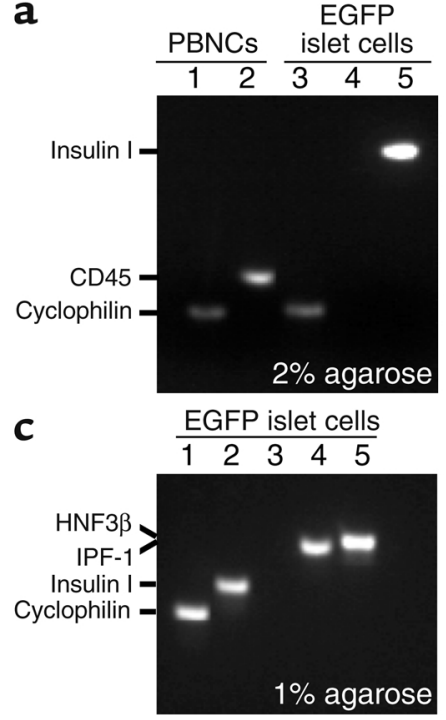

b

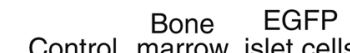
Control marrow islet cells
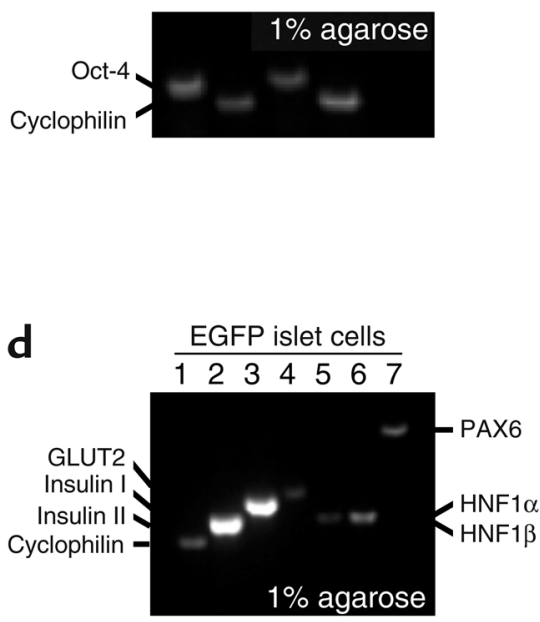


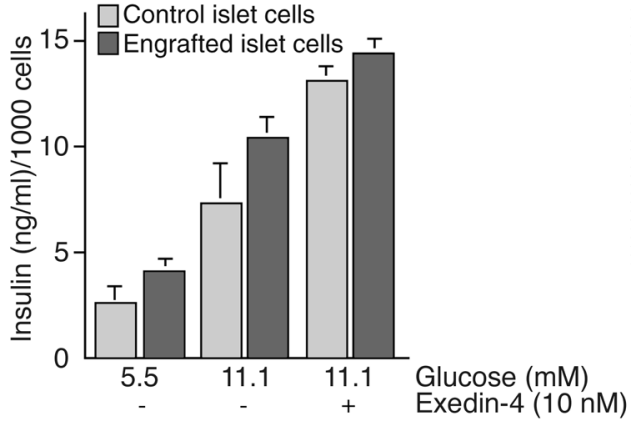

\section{Figure 6}

Insulin secretion of EGFP-positive cells isolated from islets is similar to insulin secretion of control islet cells. Insulin secretion after glucose or glucose and exendin-4 stimulation of isolated EGFP-positive cells from experiment 1 is shown as dark gray bars. Control cells were isolated from an INS*EGFP mouse and treated identically (light gray bars). One thousand dispersed islet cells were collected for EGFP expression by FACS and/or manual picking, cultured for 24 hours at $5.5 \mathrm{mM}$ glucose or $11.1 \mathrm{mM}$ glucose for 10 hours. Additional cells were incubated in $11.1 \mathrm{mM}$ glucose for 10 hours and then with 10 $\mathrm{nM}$ exendin- 4 for 4 more hours. Supernatant was collected for insulin measurements (ELISA). Results of three separate assays each performed in duplicate are shown as mean \pm SD

After sorting and culture for 24 hours, EGFP-positive cells from experiment 1 were further characterized by RT-PCR to express insulin I, insulin II, GLUT2, IPF-1, HNF1 $\alpha$, HNF1 $\beta$, HNF3 $\beta$, and PAX6, and to lack the common hematopoietic/leukocyte marker CD45 and the embryonic stem cell and bone marrow mesenchymal stem cell marker oct-3/4 (34) (Figure 5). This suggests that the bone marrow-derived cells that had engrafted the islets and that expressed EGFP did not express a non- $\beta$ cell marker present in circulating nucleated cells or in bone marrow.

EGFP-positive cells responded to glucose as well as the glucagon-like peptide- 1 synthetic analog exendin-4 (see Methods) with an insulin secretory response similar to that of control mouse islet cells that were analyzed in parallel (Figure 6). These results indicate that the cells studied have the machinery to sense glucose and the incretin hormone glucagonlike peptide- 1 similarly to wild-type mouse $\beta$ cells.

\section{Discussion}

This work demonstrates that adult mouse bone marrow harbors cells that can differentiate toward a pancreatic endocrine $\beta$ cell phenotype in recipient mice in vivo. These bone marrow-derived cells, once engrafted into the pancreatic islets of their host (Figure 2), exhibit markers and physiological behavior characteristic of pancreatic $\beta$ cells. The cells express insulin, GLUT2, and the transcription factors typically found in $\beta$ cells (Figures 3 and 5 ) and secrete insulin when stimulated with glucose or exendin-4 (Figure 6). Furthermore, these cells exhibit glucosedependent fluctuations of intracellular calcium characteristic of $\beta$ cells (data not shown).
Several previous reports have demonstrated the presence of cells within pancreatic islets $(5,11,12)$, pancreatic duct tissue $(3,9,13)$, and the liver (15) that have the potential to differentiate into cells with a pancreatic endocrine phenotype. The present report indicates an additional source of cells that are capable of differentiating into pancreatic endocrine cells. It may be hypothesized that, with regard to pancreatic islet cells, bone marrow-derived cells have the capacity to differentiate directly into insulin-producing cells. Alternatively, these cells may enter an intermediary pool of one (43) or more multipotent cell phenotype(s) within the endocrine or nonendocrine compartments of the pancreas or as oval cells within the liver (15). Then, in a further step, these cells may respond to local $(9,12,44)$ or circulating signals (45) - and differentiate into insulin-producing cells. Within the time frame of the present study (4-6 weeks), 1.7-3\% of bone marrow-derived EGFPpositive cells were detected by cell sorting in pancreatic islets. Bonner-Weir and colleagues estimate that in an adult rat islet, $3 \%$ of new $\beta$ cells are formed per day (46-48). If the turnover rate of cells in mouse islets is similar to that of rat islets, and if all newly formed $\beta$ cells are derived directly from cells originating from the transplanted bone marrow (which appears unlikely), then more EGFP-positive cells would be expected 4-6 weeks after bone marrow transplantation. However, the results of the present study do not allow this issue to be conclusively addressed. Further studies are needed to elucidate the relative contributions of resident pancreatic stem cells versus cells derived from bone marrow in the formation of new $\beta$ cells. Insulin and EGFP expression, as assessed by immunohistochemistry, was only detected within pancreatic islets. Although we detected Y chromosome-positive cells in other tissues, including the liver (not shown) and exocrine compartments of the pancreas of recipient mice (Figure 2 ), we were unable to detect any EGFP expression in cells outside of pancreatic islets. These results indicate that bone marrow-derived cells do not activate insulin gene expression unless they enter a pancreatic islet. Partial pancreatectomy (49), chronic glucose infusion $(7,50)$, and glucagon-like peptide- 1 and its analog exendin-4 (3) stimulate IPF-1 and endocrine hormone gene expression in pancreatic ducts and exocrine compartments. It would be interesting to see whether these stimuli could induce EGFP expression in the model described here.

In the present model, using the CRE-LoxP method, all cells that express insulin (and thus activate CRE and subsequent recombination events) are permanently marked with EGFP even if these cells stop expressing insulin at a later time. We have not detected EGFP-positive cells in circulating blood or in the bone marrow of successfully engrafted bone marrow recipients. Thus, it is reasonable to assume that, within the time frame of our experiments, bone marrow 
derived cells that express insulin in pancreatic islets are unlikely to leave islets and reenter the circulation, to possibly revert to a "progenitor-like" phenotype and to then populate another tissue and acquire a different phenotype in a new tissue environment (51). Alternatively, active silencing of the ROSA26-EGFP locus to turn off the green fluorescent signal would have to be postulated (52). Furthermore, the fact that we have been unable to detect EGFP-positive cells in peripheral blood or bone marrow collected from donor or recipient animals or in non-islet tissues of the recipient animals suggests (though it does not prove) that insulin gene activation with expression of CRE and subsequent disinhibition of EGFP expression do not take place before bone marrow-derived cells are within the islet.

Recent reports suggest that stem cells appear to "transdifferentiate" by cell fusion events that may lead to the detection of Y chromosome-positive cells within otherwise differentiated cells of a certain tissue (35, 36). With the CRE-LoxP system, the likelihood of fusion events can be addressed. We transplanted bone marrow from INS2-CRE mice into ROSA-stoploxEGFP recipient mice (Figure 1, experiment 2). If bone marrow-derived cells were to fuse with host cells in the islet, and the insulin II gene from the donor transgene were to be activated and express CRE, then the floxed stop codons at the ROSA26 locus in the recipient would be removed, thus rendering the cells fluorescent. While we find activation of EGFP in in vitro conditions that facilitate fusion between INS2-CRE bone marrow cells and ROSA-stoplox-EGFP islet cells (data not shown), we have not detected any fluorescence in corresponding in vivo experiments (Figures 1 and 2, experiment 2; and Figure 31). Thus, our findings suggest that cell fusion, if at all present in vivo, is a rare event in the paradigm of "transdifferentiation" of bone marrow-derived cells.

The current report demonstrates the potential for cell-based treatment of diabetes mellitus by generation of insulin-producing cells from bone marrowderived stem cells. We suggest that there are cells within the adult bone marrow that are capable of differentiating toward a pancreatic $\beta$ cell phenotype. Recently, Jiang et al. (34) and Reyes et al. (32) have described the pluripotency of mesenchymal stem cells derived from adult bone marrow that can be kept in culture for a large number of passages. Although it has not been reported (34), these cells may - as do embryonic stem cells (16) - have the capacity to differentiate into glucose-competent $\beta$ cells. If so, this strategy should allow for the isolation of cells capable of differentiating in vivo or ex vivo into functional insulin-producing cells. Furthermore, these cells might provide a potentially unlimited source of islet cells without the problems of tissue rejection as is seen in allogeneic transplantation. Apart from the therapeutic implication of these findings, the model described here may also allow examination of the mechanisms underlying the homing of cells, as well as factors controlling the regulation of gene expression, in the extramedullary environment that bone marrow-derived cells enter.

\section{Acknowledgments}

We thank D. Littman and G. Eberl for providing ROSA-stoplox-EGFP mice, T. Jessel and A. Ruiz i Altaba for the HNF3 $\beta$ antibody, and L. Dailey for the oct-3/4 control plasmid. We thank J. Hirst for help and valuable advice with fluorescence cell analysis, and D. Ron and H. Harding for enabling initial fluorescence imaging. We thank G. Eberl, J. Osbeck, and D. Ron for helpful discussions; J.A. Daly and K.N. Herman for invaluable help; and M. McAllister, M. Blaser, R. Levin, and H. Samuels for support. G.G. Holz was supported by the NIH and the American Diabetes Association (ADA), N.D. Theise by the NIH, and M.A. Hussain by the Juvenile Diabetes Research Foundation and the ADA.

1. Rosenberg, L., Vinik, A.I., Pittenger, G.L., Rafaeloff, R., and Duguid, W.P. 1996. Islet-cell regeneration in the diabetic hamster pancreas with restoration of normoglycaemia can be induced by a local growth factor(s). Diabetologia. 39:256-262.

2. Rafaeloff, R., Barlow, S.W., Rosenberg, L., and Vinik, A.I. 1995. Expression of Reg gene in the Syrian golden hamster pancreatic islet regeneration model. Diabetologia. 38:906-913.

3. Stoffers, D.A., et al. 2000. Insulinotropic glucagon-like peptide 1 agonists stimulate expression of homeodomain protein IDX-1 and increase islet size in mouse pancreas. Diabetes. 49:741-748.

4. Guz, Y., Nasir, I., and Teitelman, G. 2001. Regeneration of pancreatic beta cells from intra-islet precursor cells in an experimental model of diabetes. Endocrinology. 142:4956-4968.

5. Fernandes, A., et al. 1997. Differentiation of new insulin-producing cells is induced by injury in adult pancreatic islets. Endocrinology. 138:1750-1762.

6. Teitelman, G. 1996. Induction of beta-cell neogenesis by islet injury. Diabetes Metab. Rev. 12:91-102.

7. Lipsett, M., and Finegood, D.T. 2002. Beta-cell neogenesis during prolonged hyperglycemia in rats. Diabetes. 51:1834-1841.

8. Shapiro, A.M., et al. 2000. Islet transplantation in seven patients with type 1 diabetes mellitus using a glucocorticoid-free immunosuppressive regimen. N. Engl. J. Med. 343:230-238.

9. Bonner-Weir, S., et al. 2000. In vitro cultivation of human islets from expanded ductal tissue. Proc. Natl. Acad. Sci. U. S. A. 97:7999-8004.

10. Halvorsen, T., and Levine, F. 2001. Diabetes mellitus-cell transplantation and gene therapy approaches. Curr. Mol. Med. 1:273-286.

11. Zulewski, H., et al. 2001. Multipotential nestin-positive stem cells isolated from adult pancreatic islets differentiate ex vivo into pancreatic endocrine, exocrine, and hepatic phenotypes. Diabetes. 50:521-533.

12. Abraham, E.J., Leech, C.A., Lin, J.C., Zulewski, H., and Habener, J.F. 2002. Insulinotropic hormone glucagon-like-1 differentiation of human pancreatic islet-derived progenitor cells into insulin-producing cells. Endocrinology. 143:3152-3161.

13. Ramiya, V.K., et al. 2000. Reversal of insulin-dependent diabetes using islets generated in vitro from pancreatic stem cells. Nat. Med. 6:278-282.

14. Petersen, B.E., et al. 1999. Bone marrow as a potential source of hepatic oval cells. Science. 284:1168-1170.

15. Yang, L., et al. 2002. In vitro trans-differentiation of adult hepatic stem cells into pancreatic endocrine hormone-producing cells. Proc. Natl. Acad. Sci. U. S. A. 99:8078-8083.

16. Lumelsky, N., et al. 2001. Differentiation of embryonic stem cells to insulin-secreting structures similar to pancreatic islets. Science. 292:1389-1394.

17. Soria, B., et al. 2000. Insulin-secreting cells derived from embryonic stem cells normalize glycemia in streptozotocin-induced diabetic mice. Diabetes. 49:157-162.

18. Krause, D.S., et al. 2001. Multi-organ, multi-lineage engraftment by a single bone marrow-derived stem cell. Cell. 105:369-377.

19. Ferrari, G., et al. 1998. Muscle regeneration by bone marrow-derived myogenic progenitors. Science. 279:1528-1530.

20. Gussoni, E., et al. 1999. Dystrophin expression in the $\mathrm{mdx}$ mouse restored by stem cell transplantation. Nature. 401:390-394. 
21. Orlic, D., et al. 2001. Bone marrow cells regenerate infarcted myocardium. Nature. 410:701-705.

22. Jackson, K.A., et al. 2001. Regeneration of ischemic cardiac muscle and vascular endothelium by adult stem cells. J. Clin. Invest. 107:1395-1402.

23. Lin, Y., Weisdorf, D.J., Solovey, A., and Hebbel, R.P. 2000. Origins of circulating endothelial cells and endothelial outgrowth from blood. J. Clin. Invest. 105:71-77.

24. Asahara, T., et al. 1999. Bone marrow origin of endothelial progenitor cells responsible for postnatal vasculogenesis in physiological and pathological neovascularization. Circ. Res. 85:221-228.

25. Theise, N.D., et al. 2000. Derivation of hepatocytes from bone marrow cells in mice after radiation-induced myeloablation. Hepatology. 31:235-240.

26. Lagasse, E., et al. 2000. Purified hematopoietic stem cells can differentiate into hepatocytes in vivo. Nat. Med. 6:1229-1234.

27. Brazelton, T.R., Rossi, F.M., Keshet, G.I., and Blau, H.M. 2000. From marrow to brain: expression of neuronal phenotypes in adult mice. Science. 290:1775-1779.

28. Mezey, E., Chandross, K.J., Harta, G., Maki, R.A., and McKercher, S.R. 2000. Turning blood into brain: cells bearing neuronal antigens generated in vivo from bone marrow. Science. 290:1779-1782.

29. Theise, N.D., et al. 2000. Liver from bone marrow in humans. Hepatology. 32:11-16.

30. Korbling, M., et al. 2002. Hepatocytes and epithelial cells of donor origin in recipients of peripheral-blood stem cells. N. Engl. J. Med. 346:738-746.

31. Reyes, M., et al. 2001. Purification and ex vivo expansion of postnatal human marrow mesodermal progenitor cells. Blood. 98:2615-2625.

32. Reyes, M., et al. 2002. Origin of endothelial progenitors in human postnatal bone marrow. J. Clin. Invest. 109:337-346. doi:10.1172/ JCI200214327.

33. Schwartz, R.E., et al. 2002. Multipotent adult progenitor cells from bone marrow differentiate into functional hepatocyte-like cells. J. Clin. Invest. 109:1291-1302. doi:10.1172/JCI200215182.

34. Jiang, Y., et al. 2002. Pluripotency of mesenchymal stem cells derived from adult marrow. Nature. 418:41-49.

35. Terada, N., et al. 2002. Bone marrow cells adopt the phenotype of other cells by spontaneous cell fusion. Nature. 416:542-545.

36. Ying, Q.L., Nichols, J., Evans, E.P., and Smith, A.G. 2002. Changing potency by spontaneous fusion. Nature. 416:545-548.

37. Sauer, B., and Henderson, N. 1988. Site-specific DNA recombination in mammalian cells by the Cre recombinase of bacteriophage P1. Proc. Natl. Acad. Sci. U. S. A. 85:5166-5170.

38. Gannon, M., Shiota, C., Postic, C., Wright, C.V., and Magnuson, M.
2000. Analysis of the Cre-mediated recombination driven by rat insulin promoter in embryonic and adult mouse pancreas. Genesis. 26:139-142.

39. Soriano, P. 1999. Generalized lacZ expression with the ROSA26 Cre reporter strain. Nat. Genet. 21:70-71.

40. Mao, X., Fujiwara, Y., Chapdelaine, A., Yang, H., and Orkin, S.H. 2001. Activation of EGFP expression by Cre-mediated excision in a new ROSA26 reporter mouse strain. Blood. 97:324-326.

41. Hussain, M.A., Lee, J., Miller, C.P., and Habener, J.F. 1997. POU domain transcription factor brain 4 confers pancreatic alpha-cell-specific expression of the proglucagon gene through interaction with a novel proximal promoter G1 element. Mol. Cell. Biol. 17:7186-7194.

42. Yokoyama, W.M. 1991. Cell fusion and selection of hybridomas. In Current protocols in immunology. J.E. Coligan, A.M. Kruisbeek, D.H. Margulies, E.M. Shevach, and W. Strober, editors. John Wiley \& Sons. Hoboken, New Jersey, USA. 2.5.4-2.5.9.

43. Gu, G., Dubauskaite, J., and Melton, D.A. 2002. Direct evidence for the pancreatic lineage: NGN3+ cells are islet progenitors and are distinct from duct progenitors. Development. 129:2447-2457.

44. Czyz, J., and Wobus, A. 2001. Embryonic stem cell differentiation: the role of extracellular factors. Differentiation. 68:167-174.

45. Flier, S.N., Kulkarni, R.N., and Kahn, C.R. 2001. Evidence for a circulating islet cell growth factor in insulin-resistant states. Proc. Natl. Acad. Sci. U.S. A. 98:7475-7480.

46. Finegood, D.T., Scaglia, L., and Bonner-Weir, S. 1995. Dynamics of betacell mass in the growing rat pancreas. Estimation with a simple mathematical model. Diabetes. 44:249-256.

47. Scaglia, L., Cahill, C.J., Finegood, D.T., and Bonner-Weir, S. 1997. Apoptosis participates in the remodeling of the endocrine pancreas in the neonatal rat. Endocrinology. 138:1736-1741.

48. Bonner-Weir, S. 2001. Beta-cell turnover: its assessment and implications. Diabetes. 50(Suppl. 1):S20-S24.

49. Bonner-Weir, S., Trent, D.F., and Weir, G.C. 1983. Partial pancreatecto$m y$ in the rat and subsequent defect in glucose-induced insulin release. J. Clin. Invest. 71:1544-1553.

50. Jonas, J.C., et al. 1999. Chronic hyperglycemia triggers loss of pancreatic beta cell differentiation in an animal model of diabetes. J. Biol. Chem. 274:14112-14121.

51. Theise, N.D., and Krause, D.S. 2002. Toward a new paradigm of cell plasticity. Lenkemia. 16:542-548.

52. Trainor, P.A., et al. 1999. Application of lacZ transgenic mice to cell lineage studies. In Molecular embryology: methods and protocols. Volume 97. P.T. Sharpe and I. Mason, editors. Humana Press. Totowa, New Jersey, USA $183-200$ 